\title{
Investigation of the dynamical characteristics of the lower-limbs exoskeleton actuators
}

\author{
Andrey Yatsun $^{1, *}$, Andrey Karlov $^{1}$, Andrey Malchikov ${ }^{1}$ and Sergey Jatsun ${ }^{2}$ \\ ${ }^{1}$ South-West State University, Robotics Department, 305040, 50 let Oktyabrya, 94, Kursk, Russia \\ ${ }^{2}$ Mechanical Engineering Research Institute of the Russian Academy of Sciences, 101990, Moscow, Russia
}

\begin{abstract}
Authors present results of the theoretical modeling and experimental tests of the low-cost DCmotors, used in lower limb powered exoskeleton. Actuators work in difficult regime and it is important to achieve desired parameters, even for not robust motors. Results give us information and methods and means of experimental determination of the main characteristics of the robot's actuators. It gives possibility to tune control system and the whole system to achieve optimal walking regime.
\end{abstract}

\section{Introduction}

Nowadays robotic lower-limbs exoskeletons are commonly used for solving many problems, from reducing the heavy load on the human body during walking, up to the restoration of the locomotion abilities of injury patients $[1,2]$. One of the key challenges in developing such devices is the synthesis and tuning of control system parameters, providing high precision movement of an exoskeleton parts in the presence of different disturbances, also taking into account the properties of the actuators [3, 4].

Non-linear characteristics of the actuator are one of the problems associated with accurate control of multilink electromechanical systems. In particular, the actuator is characterized by the saturation of maximum torque and the presence of dead zone due to reducer. The saturation of the torque indicates that the drive is not able to produce a torque greater than some limit value, regardless of the strength of his signal. The effect of dead zones based on some range of values supplied to the drive signal at which the drive produces no torque.

In [7] indicates that the nonlinearity of the actuator has a greater impact on the quality of the control systems than the uncertainty in the model parameters of the mechanical system that was used in the design and configuration of the automatic control system. One of the most significant problems caused by the nonlinearity of the actuators as precision mechanical systems, is the controller windup. This effect is characteristic for a servo linear control systems feedback, especially for PID controllers. It based on the fact that the regulator requires from the drive more time than one can generate, therefore there is a growing error in the position tracking system. This leads to a change in the behavior of the control system, occurrence of overshoot and oscillations. For high-precision control systems, e.g. control systems of multi-link mobile robots, such effects are unacceptable.
To eliminate these problems, we tune controller in different approaches. Among the most common increase the differential component of the PID controller, the scaling of the nominal exposure.

The aim of this work is to develop methods and means of experimental determination of the main characteristics of the actuator used in the creation of exoskeletons.

\section{Experimental setup}

To achieve this goal experimental setup have been developed and constructed for the study of the characteristics of the actuators. Figure 1 shows the model system for the study of the characteristics of the wiring.

The experimental setup consists of the following main parts: 1 - clamping chuck for motor's shafts with diameters from 3 to $16 \mathrm{~mm} ; 2$ - a shaft transmitting rotation from the output shaft of the electric drive; 3 rotational disc with a large diameter; 4 - friction pair; 5 - rotation disk; 6 - pressing spring; 7 - rear carriage; 8 - the handle to move the carriage; 9 - basement; 10 feedback optical sensor; 11 - gauge efforts; 12 - thrust bearing; 13 - front carriage; 14 - adjusting screw.

The device operates as follows. The power source control used to set the required rotation speed. After power on, motor with gearing, by the clutch drives to the measuring disc and the friction drive plate. Using the current meter and phototachometer, is determined by current consumption and the frequency of rotation of the drive shaft. Next, using the handle, the preload spring of the friction drive element pushes to the leading. On the contact surface of friction disks, the friction force leads to the displacement of the disk. Disk is connected via an adjustable rod, a spring-loaded force gauge, prevents the rotation of the driven disk. Thus, knowing the magnitude of the force $F$, acting from the drive element and knowing the rod length $L$, 
we can estimate the friction torque $M c$ that occurs between the discs. Using the software signal is processed and plotted to graphs.

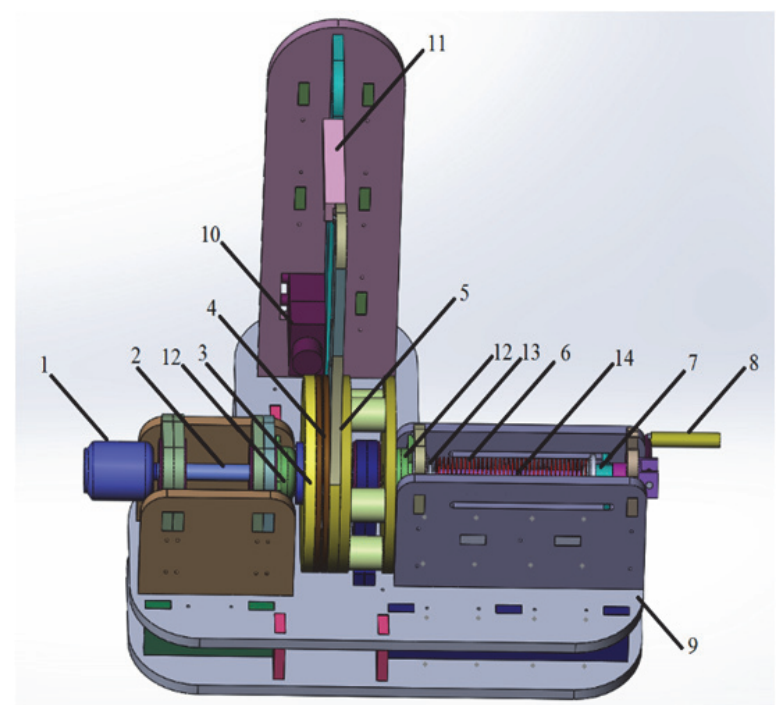

Fig. 1. General view of the experimental setup to study the characteristics of the actuator.

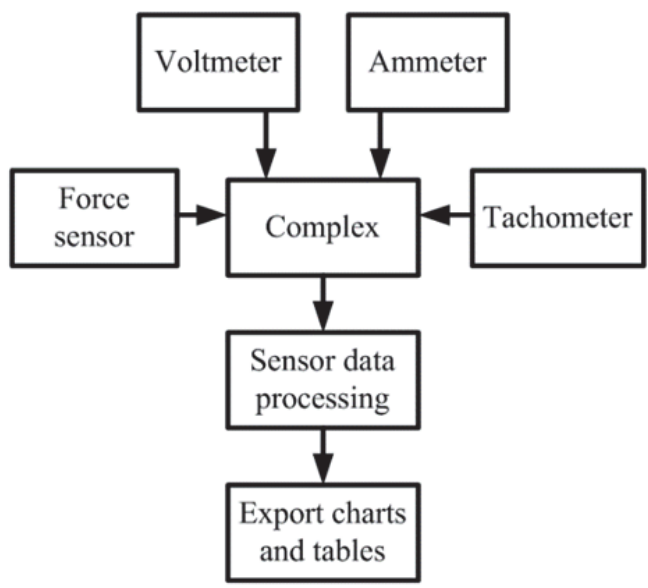

Fig. 2 Structural scheme of automated complex to study the characteristics of the actuator.

\section{Comparative analysis of the actuators}

On the basis of data obtained in an analytical way, the actuators of the lower limbs exoskeleton with a standing person should create torque al least $25 \mathrm{Nm}$ and achieve rotation speed up to $1.8 \mathrm{rad} / \mathrm{sec}$. Modern international companies offer brushed and brushless motors with similar characteristics but their products have high price (at least 150 \$) [6]. So, we will investigate low-cost electric drive with similar characteristics for acquisition of the exoskeleton.

We offer brushed DC-motor DSH-12RSH3 with 3speed planetary gearbox with price less than $20 \$$ each. Nominal voltage is 12-14 Volts, and idle speed is 16000 $\mathrm{rpm}$. The motor diameter is $38 \mathrm{~mm}$, the length is $74 \mathrm{~mm}$, dimensions of gear is $50 \times 43 \mathrm{~mm}$, length is $97 \mathrm{~mm}$. the total length of the assembly is $160 \mathrm{~mm}$, weight is 475 grams (Fig.3).

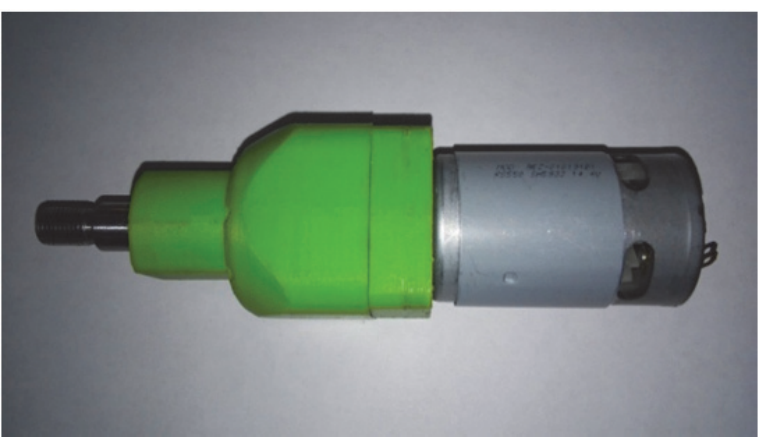

Fig. 3a Photo of the low-cost gear motor DSH-12RSH3.

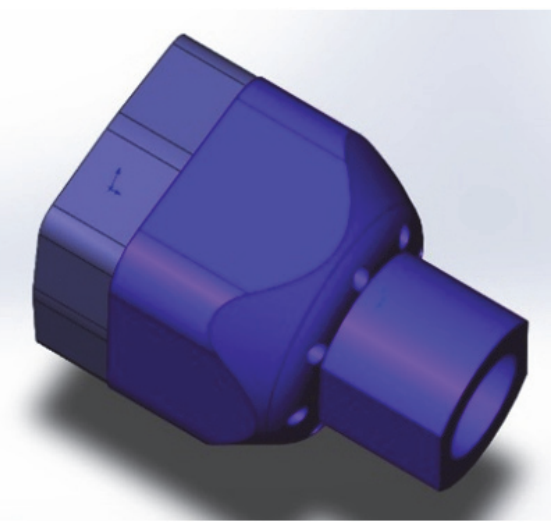

Fig. 3b The 3D model of the 3 stages planetary gear box RSH3 own development.

The planetary gearbox has its own design, consists of 3 stages, gear ratio 6 each, so total reduction ratio is 206 . Using CAD-system to study the characteristics of the actuator we have received a table of values for different supply voltage and has built a family of mechanical characteristics for this drive.

According to tests maximum torque is $16 \mathrm{Nm}$, and the maximum angular speed is equal to $7,98 \mathrm{rad} / \mathrm{s}$ at 12 Volts supply voltage. This drive does not allow to develop the effort to provide the speed necessary for the verticalization of the person in the exoskeleton (Fig.4).

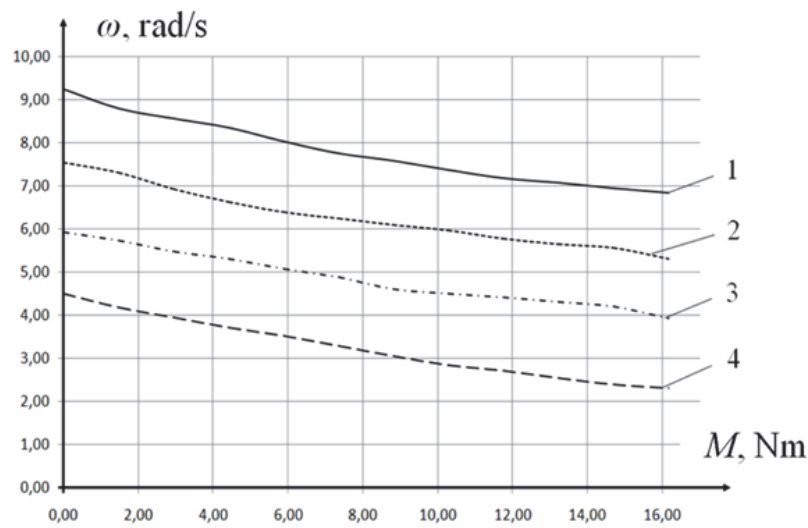

Fig. 4. The experimental family plots of mechanical characteristics DSH-12RSH3: 1 is for 12 Volts; 2 is for 10 Volts; 3 is for 8 Volts; 4 is for 6 Volts.

In connection with these experimental results it is necessary to add another gear stage that allows us to achieve maximum torque not less than $25 \mathrm{Nm}$ on the 
output shaft with the angular velocity $1.8(\mathrm{rad} / \mathrm{s})$. So we developed brushed DC-motor DSH-12RSH4 with additional 4-th stage (Fig.5)

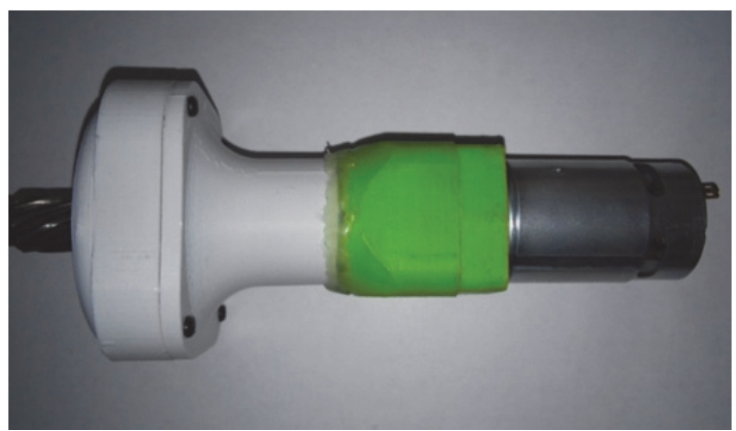

Fig. 5a. Photo of the low-cost gear motor DSH-12RSH4.

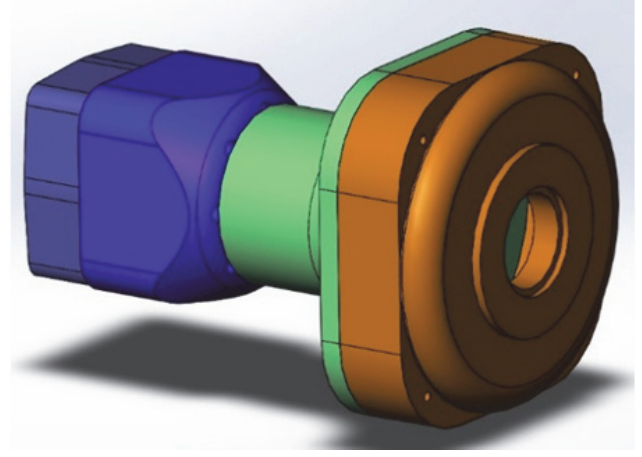

Fig. 5b. The 3D model of the 3 stages planetary gear box RSH4 own development.

Actuator called DSH-12RSH4 with a planetary 4stages gearbox has such dimensions: gearbox size $72 \times 72 \mathrm{~mm}$, gear length is $130 \mathrm{~mm}$, total assembly length is equal to $200 \mathrm{~mm}$, weight is $870 \mathrm{gr}$.

The planetary gear consists of 4 steps, based on the gearbox RSH3, and the fourth with a gear ratio of 4.15. Total reduction ratio is 855 . Family of actuator mechanical characteristics are shown in Figure 6.

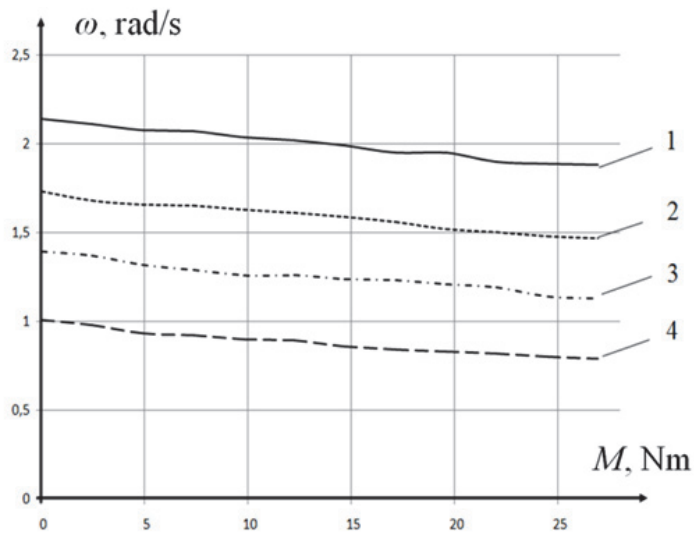

Fig. 6. The experimental family plots of mechanical characteristics DSH-12RSH2: 1 is for 12 Volts; 2 is for 10 Volts; 3 is for 8 Volts; 4 is for 6 Volts.

Maximum torque is $27 \mathrm{Nm}$, the maximum angular speed equal to $1.9 \mathrm{rad} / \mathrm{s}$ at 12 Volts supply voltage. This corresponds to the desired torque and speed characteristics of the actuator.
Further we investigate dynamical parameters of brushed DC-motor DSH-18, which nominal supply is 18 Volts and idle speed 19000 rpm with two-stage RSH2 and three-stage RSH3 planetary reducers (Fig. 7-9).

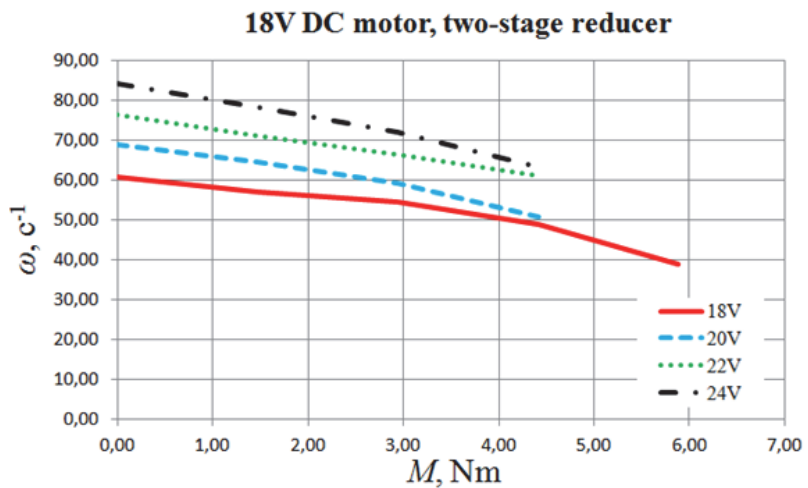

Fig. 7. The experimental family plots of mechanical characteristics DSH-18RSH2.

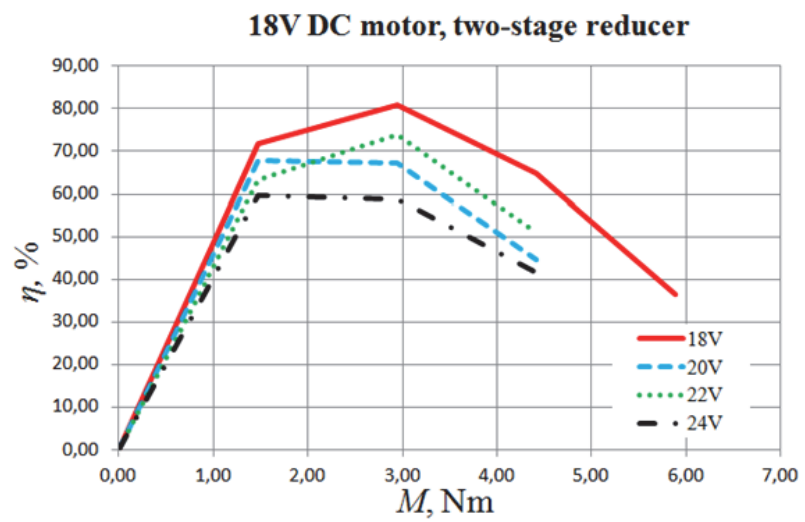

Fig. 8. The experimental efficiency for DSH-12RSH2 actuator.

The graphs show that the rotation speed and torque below the required parameters, but this can be compensated with the use of ball screw drive (linear actuator).

$\omega, \mathrm{rad} / \mathrm{s} \quad 18 \mathrm{~V}$ DC motor, two-stage reducer

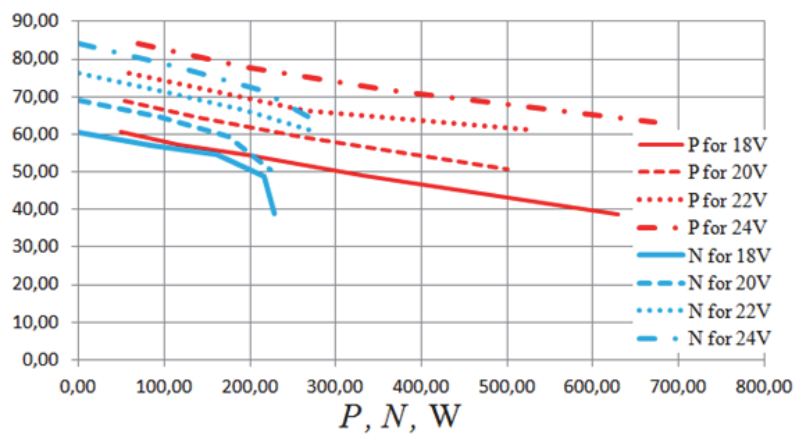

Fig. 9 Experimental graph of power in dependence on frequency of rotation: $\mathrm{P}$ - electrical power, $\mathrm{N}$ - mechanical power.

On the next experiment we get information about brushed DC-motor assembly DSH-18RSH3 (Fig. 10-12). 
18V DC motor, tree-stage reducer

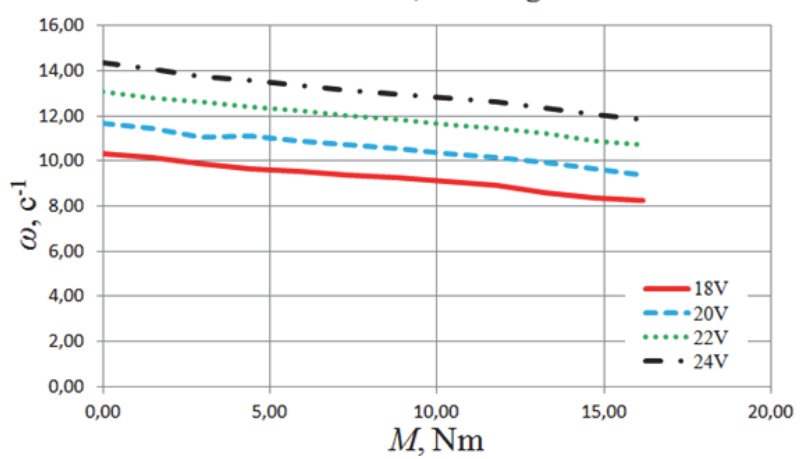

Fig. 10. The experimental family plots of mechanical characteristics DSH-18RSH3.

18V DC motor, tree-stage reducer

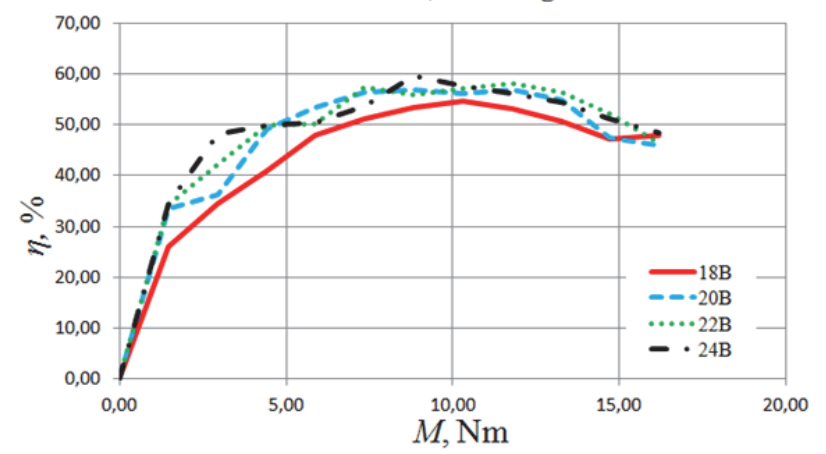

Fig. 11. The experimental efficiency for DSH-12RSH3 actuator

18V DC motor, three-stage reducer

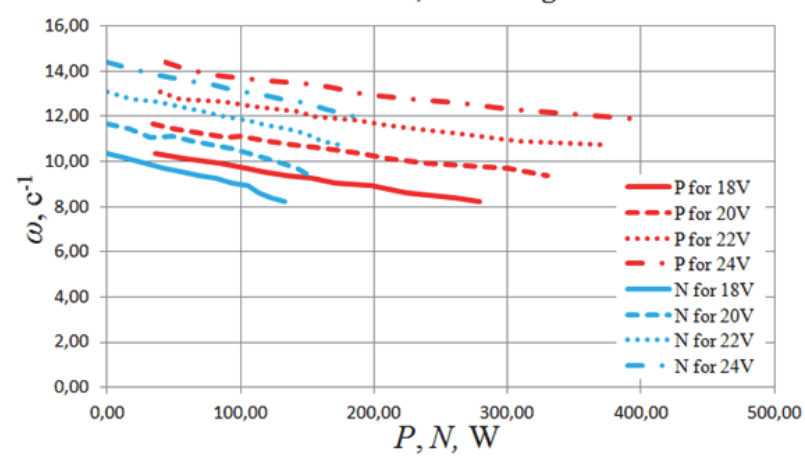

Fig. 12. Experimental graph of power in dependence on frequency of rotation: $\mathrm{P}$ - electrical power, $\mathrm{N}$ - mechanical power.

According to these results we can obtain optimal parameters of the assembly for the prototype of the lowerlimb exoskeleton or bipedal walking robot. At figure 13 presents photo of the exoskeleton prototype with DSH12RSH4 actuators, which was developed at the Robotics Laboratory of South-West State University (Kursk).

\section{Analysis of the electric current in the windings}

For the accurate control of the output torque it is important to get filtered feedback current into the controller. Due to real-time approximation, based on microcontroller we used such ways: a linear fit of the experimental data using the least squares method, linear approximation passing through the start and end points, logarithmic approximation passing through the start and end points.

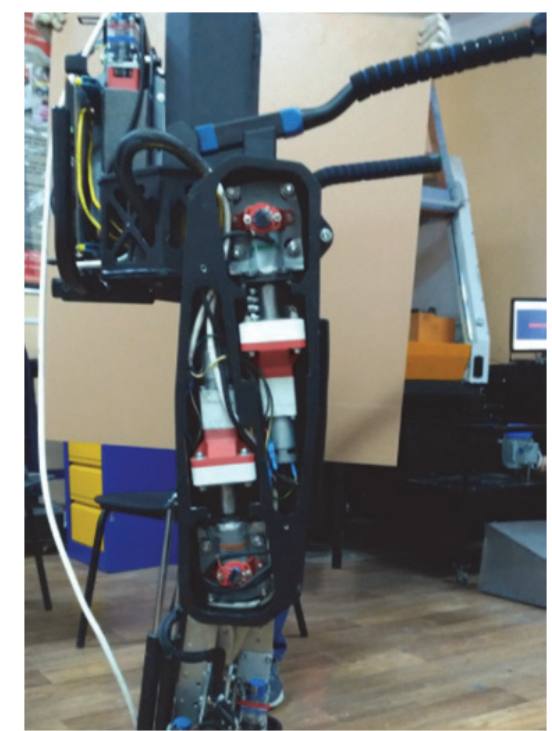

Fig. 13. Exoskeleton prototype with DSH-12RSH4 actuators.

The original data and the resulting linear approximation for the winding current of the DSH12RSH3 at upper limit 22 Volts power supply is shown in Figure 14.

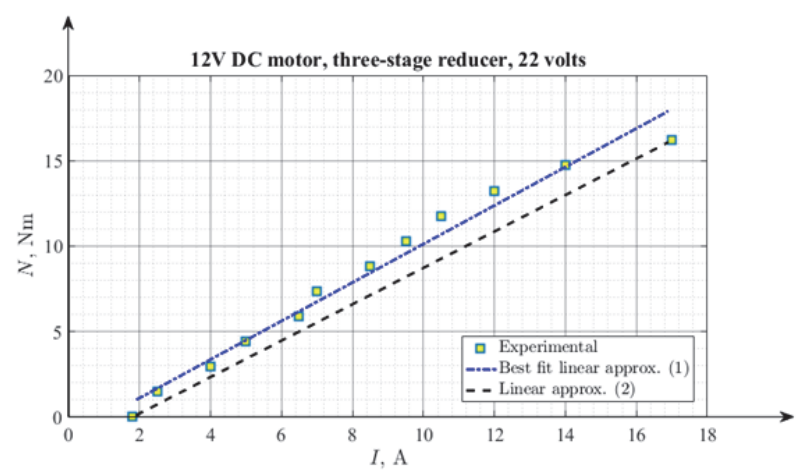

Fig. 14. Experimental data and linear fit for DSH-12RSH3.

The logarithmic approximation of the experimental data passing through the start and end points of the data is more curving. The original data and the resulting approximation for the winding current of the DSH$18 \mathrm{RSH}$ at 20 Volts power supply is shown in figure 15.

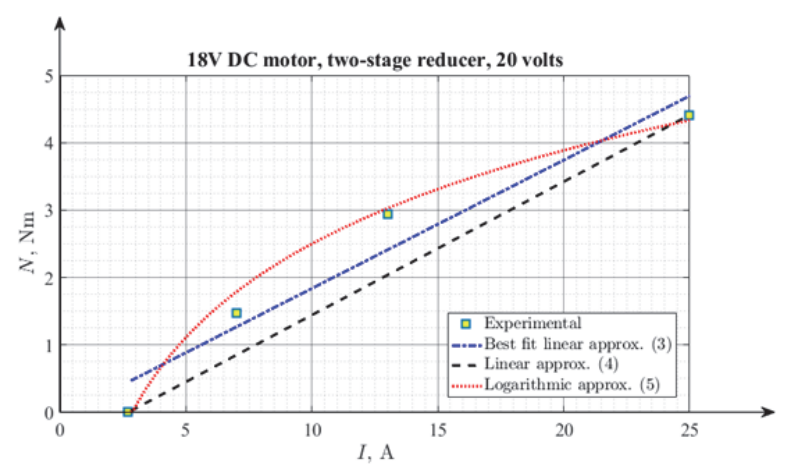

Fig. 15. A linear fit of the experimental data for DSH-18RSH2. 
Thus, the torque of the actuator from current is described by the formulae:

$$
\left\{\begin{array}{l}
M=\operatorname{sign}(I) \cdot(2 \log (|I|)+2.5) \quad \text { if } \quad|I|>2.7 \\
M=0 \quad \text { if } \quad|I| \leq 2.7
\end{array}\right.
$$

We will consider the motor parameters $L=0.001 \mathrm{H}$, $R=0.5 \mathrm{Ohm}, C_{e}=0.0033, C_{\tau}=0.0033, N=100$ and will conduct numerical experiments for the following values of the controller parameters:

$$
k_{p}=500, k_{d}=20, A_{p}=20 .
$$

At figure 16 we obtain results of modelling : desired and real angular speed of the motor shaft depending on time, which corresponds to DSH-12RSH3 with torque feedback control.

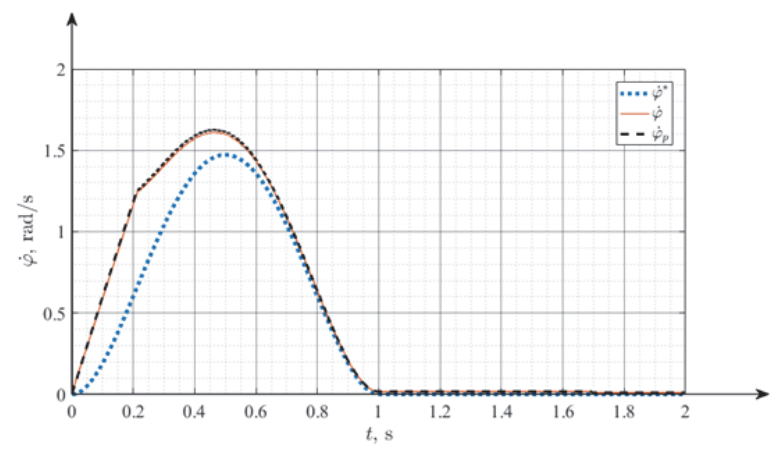

Fig. 16 Angular speed time dependence for actuator $12 \mathrm{~V}$ with a three-stage reducer.

This graph is comparable with that obtained in experimental part, one can notice that the maximum torque developed by the actuator, which in this case was $5.5 \mathrm{Nm}$, which was not taken into account the experimental dependence of the torque of a current, this value was $8.5 \mathrm{Nm}$.

The obtained simulation results (without taking into account the experimental data on the dependence), show that the steady-state value of the current in the windings of the motor is $26 \mathrm{~A}$. In this case, the steady-state current value was $44 \mathrm{~A}$, i.e. $69 \%$ more. The steady-state value of the voltage was 12.5 , i.e. $80 \%$ more. Static error in this case has decreased significantly.

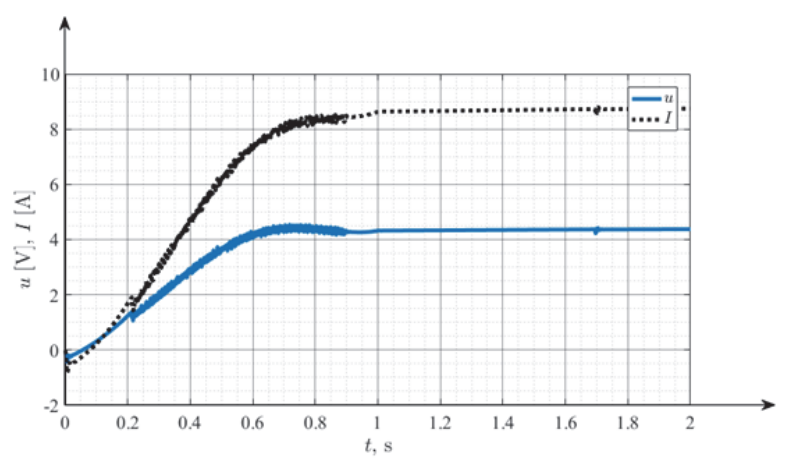

Fig. 17. Time dependence of supply voltage and current; used the model corresponding actuator $12 \mathrm{~V}$ with a three-stage reducer.
In this case the steady-state current value amounted to $8.7 \mathrm{~A}$, i.e. $66 \%$ less than in the experiments. The steady-state value of the voltage was $4.5 \mathrm{~V}$, i.e. $64 \%$ less than in the data section

\section{Conclusions}

Description of the experimental stand, on which the various tests developed for electric drives is presented. The experiments show that the optimal design of an Assembly for use in walking robots and exoskeletons. The use of experimental data allows to confirm the simulation results and to determine the optimal coefficients of the controller for a feedback system in which the currents do not exceed the level of permissible values. According to the modeling results we obtained transfer characteristics for the control system.

This research is supported by grant of the RF President for young scientists MK-2701.2017.8.

\section{References}

1. J. Zhang, P. et. al., Science, 356 (6344), 1280-1284 (2017)

2. M. Sczesny-Kaiser et. al. Frontiers in Neuroscience, 11, 449 p. (2017).

3. A.P.P.A. Majeed et al, Procedia Computer Science, 105, 183-190 (2017)

4. G.Ya. Panovko, S.F. Jatsun, S.I. Savin, A.S. Jatsun, Machinery and engineering education, 2 (47), 2-10 (2016)

5. A.J. Young, J. Foss, H. Gannon, and D.P. Ferris, Frontiers in Bioengineering and Biotechnology, 5, 4 (2017)

6. S. Jatsun, S. Savin, A. Yatsun, 24th Mediterranean Conference on Control and Automation (MED), 322-326 (2016)

7. F. Tyan, D.S. Bernstein, Journal of Robust and Nonlinear Control, 5 (5), 521-537 (1995)

8. S. Jatsun, 2015 10th International Symposium on Mechatronics and its Applications (ISMA), 1-6 (2015)

9. S. Jatsun, Proceedings of the International Conference on Pure Mathematics - Applied Mathematics, 83-87 (2015)

10. S. Jatsun, Proceedings of the 24th International Conference on Robotics in Alpe-Adria-Danube Region (RAAD), 371, 165-172 (2015) 